\title{
Assessing the role of body mass and sex on apparent adult survival in polygynous passerines: a case study of cetti's warblers in central Portugal
}

\author{
David Monticelli, Pedro M. Araújo, James E. Hines, Paulo Q. Tenreiro, Luis P. Silva \\ and Jaime A. Ramos
}

D. Monticelli (monticelli.david@gmail.com), P. M. Araújo, L. P. Silva and Jaime A. Ramos, Dept of Life Sciences, Univ. of Coimbra, Apartado 3046, PT-3001-401 Coimbra, Portugal. - J. E. Hines, US Geological Survey, Patuxent Wildlife Research Center, 12100 Beech Forest Road, Laurel, MD 20708, USA. - P. Q. Tenreiro, Inst. para a Conservação da Natureza e das Florestas (ICNF), Reserva Natural do Paul de Arzila, Rua do Bairro 1, PT-3045-356 Arzila, Portugal.

\begin{abstract}
Adult survival, an important fitness component, is usually 1) lower in lighter individuals due to their reduced ability to survive winter conditions compared to heavier ones, especially in resident species at northern temperate latitudes and 2) lower in females compared with males due to higher reproductive costs incurred by females. In this paper, a capture-mark-recapture dataset of 649 cetti's warblers Cettia cetti ringed seasonally at two wetlands in central Portugal over an 11-yr period (2000-2010) was modelled in a multi-state framework to examine the influence of these individual covariates on apparent adult survival, while controlling for the presence of transient individuals in our study area. The probability of change in mass state $\left(\psi_{\text {Light } \rightarrow \text { Heavy }}, \psi_{\text {Heavy } \rightarrow \text { Light }}\right)$ during the annual cycle was also estimated. Overall, birds survived better during spring-summer (breeding/moulting periods) compared with autumn-winter, but there was no effect of body mass on apparent adult survival probability. The modelling detected a significant interaction between sex and season, in which resident females survived better than resident males in springsummer $\left(\phi_{\mathrm{RF}}=0.857 \pm 0.117\right.$ and $\left.\phi_{\mathrm{RM}}=0.698 \pm 0.181\right)$ while the opposite pattern was found in autumn-winter $\left(\phi_{\mathrm{RM}}=0.440 \pm 0.086\right.$ and $\left.\phi_{\mathrm{RF}}=0.339 \pm 0.084\right)$. In addition, cetti's warblers had a tendency to lose mass in springsummer $\left(\psi_{\text {Heavy } \rightarrow \text { Light }}=0.560 \pm 0.063\right)$ and to regain mass in autumn-winter $\left(\psi_{\text {Light } \rightarrow \text { Heavy }}=0.701 \pm 0.069\right)$. This pattern of body mass change during the annual cycle may reflect energetic costs to reproduction and moulting, and/or a response to increased starvation risk during winter. High body mass, however, did not increase adult survival in this population presumably due to the relatively mild winter weather prevailing in central Portugal. Survival estimates are more likely to be explained by important ecological and behavioural differences between the two sexes in polygynous passerines. Our results highlight that studies aiming to identify the main factors shaping survival and individual fitness in polygynous species should be conducted during different phases of their annual cycle.
\end{abstract}

Survival is an important fitness component playing a central role in animal population dynamics, and understanding the factors that affect it is important in population demography and evolutionary ecology studies. While environmental conditions such as weather and food availability (Peach et al. 1999, Robinson et al. 2007a, Bocci et al. 2010), and density-dependent processes (Tinbergen et al. 1985, Lieske et al. 2000, Sæther et al. 2002, Bocci et al. 2010), are key factors shaping survival and hence population demography, individuals may be differently affected by these factors, depending on their particular attributes within a given population. For example, survival may be related to age (Clobert et al. 1988), sex (Post and Götmark 2006a), or may vary between individuals according to their social status (Lundberg 1995, Schubert et al. 2008).

In birds, particularly passerines, body mass is also an important determinant of fitness and survival of individuals
(Houston and McNamara 1993, Covas et al. 2002). For example, post-fledging survival and local recruitment rates are often positively correlated to body mass at fledging (Naef-Daenzer et al. 2001). There is also a positive relationship between adult survival and body mass, especially in resident populations at northern temperate latitudes, where the lightest individuals are those that carry low fat reserves and have thus reduced chances to survive during the cold winter months (Houston and McNamara 1993, Gosler 1996). However, high body mass has also survival costs presumably because the advantage conferred by carrying considerable amounts of fat reserves may be offset, at least for the heaviest individuals, by increased predation risk due to reduced flight efficiency (Covas et al. 2002). As a result, birds might optimise their survival prospects by maintaining their body mass and fat reserves at a lower level than that of which they are capable (Lima 1986, McNamara and Houston 1990, Witter and Cuthill 1993). 
Understanding the patterns of changes in body mass during the annual cycle of passerine species has also proved useful to unravel important aspects of their life history strategies (Moreno 1989). Empirical studies have shown that body mass usually increases during autumn and winter to reach a peak in mid-winter when the energetic requirements are highest (Cresswell 1998, Macleod et al. 2005). Conversely, adults are often prone to lose body mass during nesting (Newton 1998, Boyle et al. 2012), especially females (Freed 1981) due to their higher parental investment (egglaying, incubation, nestling feeding) compared with males (reviewed by Donald 2007).

Most studies examining the effect of body mass on the survival of songbirds are nevertheless based on monogamously breeding species. Extra-pair copulation and occasional cases of bigamy and polygyny are not rare in many species (Birkhead and Møller 1995, Pilastro et al. 2002), and regular polygyny has been documented in 5\% of species in both North American and European passerines. A peculiar trait of polygynous species is the size-dimorphism between the sexes, with males being larger than females (Searcy and Yasukawa 1981). In addition, polygynouslymated females incubate alone and male involvement in paternal care of offspring is dramatically reduced (Bibby 1982, Webster 1991). Studies examining the relationship between body mass, sex and adult survival have been previously conducted with several polygynous species of the new world family Icteridae (Johnson et al. 1980, Searcy and Yasukawa 1981). However, the 'survival' estimates obtained and discussed in those studies actually referred to ad hoc return rates of ringed individuals and thus did not account for the potential bias introduced by unequal detection (recapture) probabilities frequently found between individuals or groups of individuals (e.g. males and females; Amrhein et al. 2012). The risk of flawed inference from capture-mark-recapture (CMR) datasets can be reduced by relying on modern modelling techniques, which formally account for imperfect detection probabilities (Gimenez et al. 2008). For instance, multi-state mark-recapture models can be tailored to investigate the relationships between individual state variables and survival probability, making them of great interest to address questions in evolutionary ecology (Nichols and Kendall 1995). Body mass, a proxy measure of individual fitness under selection pressure in bird populations (Covas et al. 2002), is one example of such dynamic individual state variable that can be readily analysed in a multi-state framework (Nichols et al. 1992). To our knowledge, however, very few studies have modelled mark-recapture data with body mass used as a state variable (but see Miller et al. 2003, McGowan et al. 2011), despite the fact that national ringing scheme procedures usually incorporate the collection of mass data and/or fat scores at each capture-recapture of individual birds (EURING 2010).

In the present study, we analysed a 11-yr CMR dataset on the cetti's warbler Cettia cetti, a regulary polygynous species resident in most of southwestern Europe. Multi-state models (Nichols et al. 1992) with body mass (categorized into discrete mass classes) used as the individual state variable were developed to test the following hypotheses.
1) According to the starvation-predation risk tradeoff, mass gain is adjusted during the annual cycle in order to maximise survival. Specifically, we expected individuals to (1.1.) gain mass during the winter (non-breeding) season in order to minimize the risk of starvation (McNamara and Houston 1990, Macleod et al. 2005) and (1.2.) to lose mass, especially females, during the breeding season (Freed 1981, Boyle et al. 2012).

2) Apparent adult survival might be lower during the winter season (i.e. compared to summer), with those individuals with low body mass (and therefore low fat reserves) exhibiting lower survival rates than heavier ones (Newton 1998).

3) A priori information on the peculiar ecology of this polygynous species suggests that females incubate eggs and raise their chicks alone, while males spend most of their time advertising and defending large territories during breeding. Previous studies on monogamous species with a strong parental investment of females compared with males found a strong reproductive cost for the former (i.e. due to high predation rate on females; Post and Götmark 2006a). Alternatively, previous studies in a few polygynous (non-passerine) bird species (Angelstam 1984) reported significant mating costs lowering adult male survival. We thus expected that marked behavioural differences between male and female cetti's warblers, particularly during the breeding season, may lead to sex-specific reproductive costs and adult survival rates.

\section{Material and methods}

\section{Study species and area}

The cetti's warbler Cettia cetti is a largely sedentary, insectivorous passerine associated with dense and tangled shrubby vegetation growing near water (Cramp and Brooks 1992). It is the sole European warbler species with a pronounced sexual size dimorphism and one of the few with a polygynous breeding system (Bibby and Thomas 1984). On average, males are bigger than females by ca $26-32 \%$ in body mass and ca $11-13 \%$ in wing-length with minimal overlap between the sexes (Bibby 1982, Bibby and Thomas 1984). During the breeding season, which lasts from March to early July, males hold large territories and can breed simultaneously with up to three females (Bibby 1982). Females take care of the incubation alone and provide most of the feeding to the nestlings (Bibby 1982). In late June-early July, adults start a post-breeding moult completed in ca $60 \mathrm{~d}$ (Bibby and Thomas 1984).

The capture-mark-recapture (CMR) sessions were conducted at two wetland marshes, Paul do Taipal and Paul da Madriz, west of Coimbra, central Portugal. The two sites are classified as special protection areas (SPAs) and are part of a large network of wetlands stretching along the Mondego valley. Paul do Taipal $\left(40^{\circ} 11^{\prime} \mathrm{N}, 8^{\circ} 41^{\prime} \mathrm{W}\right)$ (233 ha), is a densely vegetated marsh resulting from the permanent flooding of long-abandoned rice fields. It supports a wide variety of breeding water birds and is of national importance for wintering ducks, as well as an important stop-over site for migrating passerines. Paul da Madriz $\left(40^{\circ} 7^{\prime} \mathrm{N}, 8^{\circ} 38^{\prime} \mathrm{W}\right)$ 
is located ca $12 \mathrm{~km}$ to the south of Taipal and is a smaller freshwater marsh (89 ha) surrounded by mixed pineoak woodland and agricultural fields. At both sites, the dominant vegetation near water consists of reed Phragmites australis and bulrush Scirpus lacustres.

\section{Mist-netting protocol}

From February 2000 to November 2010 (11 yr), cetti's warblers were captured (mist-netted) and re-trapped at these two sites as part of a larger project including the study of reed warblers Acrocephalus scirpaceus and other migratory passerines. CMR sessions were conducted monthly (range 1-6 ringing sessions/month). During the breeding season (March-July), the mist-netting protocol followed the guidelines of the Portuguese constant effort sites scheme (PEEC), as part of a European project (Euro-CES: Constant effort ringing in Europe) promoted by EURING.

\section{Ageing and sexing birds}

At first capture, cetti's warblers were ringed with a metal leg ring provided by the Portuguese Central Ringing Office and aged as either first-year (juvenile) or adult according to plumage characteristics (Svensson 1992). Maximum wing length was measured to the nearest $0.5 \mathrm{~mm}$ and the birds were weighed to the nearest $0.1 \mathrm{~g}$ with an electronic balance. Wing length and body mass were controlled at any subsequent recapture. Previous work in UK (Bibby 1982), Spain (Villaran 2000) and Portugal (Bibby and Thomas 1984) found a strong size dimorphism in the cetti's warbler with females being shorter-winged and lighter than males. Based on the subset of birds caught with a brood patch, those individuals that had wing length $\leq 59 \mathrm{~mm}$ and were lighter than $12.0 \mathrm{~g}$ were sexed as females while those individuals with body mass $\geq 12.0 \mathrm{~g}$ and wing length $\geq 60 \mathrm{~mm}$ were sexed as males. A minority of individuals (ca 3\%), mainly juve- niles, did not comply with both criteria and hence could not be confidently sexed. This might be due to the fact that juvenile males can have slightly shorter wings compared to adults (i.e. on average $1.6 \mathrm{~mm}$ shorter; Bibby and Thomas 1984) and/or because wing length measurement in the field is subject to error. In addition, it should be acknowledged that a degree of uncertainty, albeit small, existed when assigning sex for both adults and juveniles solely based on body mass and wing length measurements. Thus, intermediate birds with 12.0 to $12.4 \mathrm{~g}$ in mass and/or wing length of 58 to 59 $\mathrm{mm}$ were removed from the modelling in order to minimize the odds of incorporating misclassified cetti's warblers with respect to sex.

\section{Capture-recapture sampling periods}

Despite a near-constant monthly mist-netting effort during the study period, one of the underlying assumptions of CMR models is that the recapture occasions are of negligible length relative to the time interval between them (Williams et al. 2002). In order to select discrete capturerecapture sampling periods, the capture-recapture history dataset was plotted on a monthly basis (Fig. 1). For each year, a peak in capture-recaptures was apparent during the spring and autumn seasons. We thus opted for two annual recapture periods, 1 March to 30 April (spring) and 1 September to 30 October (autumn), for a total of 22 sampling periods from spring 2000 to autumn 2010. The recapture data obtained outside the sampling periods were not incorporated in the analysis and for birds that were caught multiple times during a recapture period, only one record/period was used during the modelling. Individuals were considered to survive between the mid-points of two successive recapture occasions, which corresponds to a six-month survival rate estimated separately for the breeding/moulting season (1 April-30 Sept) and the post-

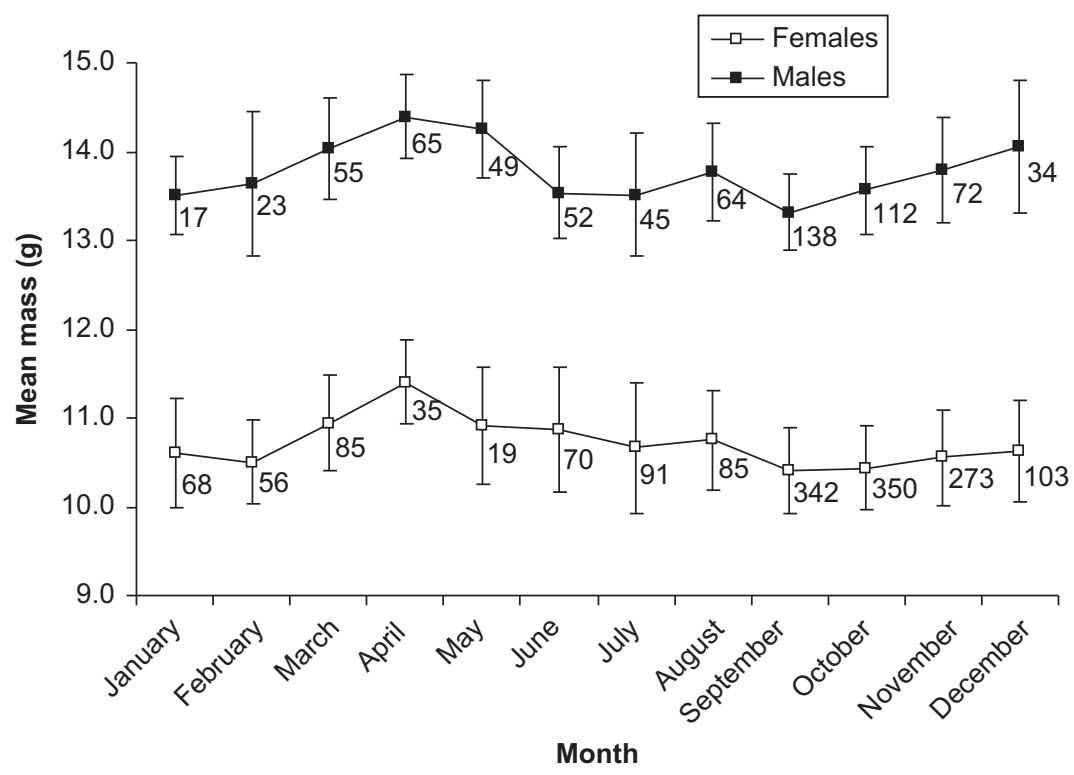

Figure 1. Annual cycle of variation in mean mass $( \pm$ SE) of male and female cetti's warblers mist-netted in central Portugal, $2000-2010$. Sample sizes of monthly capture-recaptures are indicated below each respective plot labels. Data are shown separately for the subset of females $(9.0-11.9 \mathrm{~g})$ and males $(12.0-16.0 \mathrm{~g})$. 
moulting/non-breeding season (1 October-30 March). Importantly, the terminology 'survival' used in this paper refer to an apparent survival $\phi$, i.e. the probability of surviving between two successive sampling periods and remaining in the study area. As a result, the counterpart of 'survival' includes both mortality and permanent emigration from our study area (Williams et al. 2002).

\section{CMR data modelling}

Multi-state capture-recapture models (Lebreton et al. 2009) were fitted using body mass class as a (stochastic) state variable (Nichols et al. 1992), in which individuals were assigned to one of the following states: light female (9.0-10.4 g), heavy female (10.5-11.9 g), light male $(12.5-13.9 \mathrm{~g})$ and heavy male $(14.0-16.0 \mathrm{~g})$. When individuals were capturerecaptured multiple times during a sampling period, the assignment to a mass class for that particular sampling period was made based on the mean mass measurement. Based on the assignment of the birds at each capture-recapture occasion as either light or heavy individuals, we modelled three kinds of parameters: apparent survival $(\phi)$, probability of change in, or movement between, mass class ( $\psi$; state transition probability) and capture probability (p). During the modelling, transitions between mass states $\left(\psi_{\text {Light } \rightarrow \text { Heavy }}\right)$ and $\left(\psi_{\text {Heavy } \rightarrow \text { Light }}\right)$ were only permitted within each sex.

European cetti's warbler populations are resident, although adults may disperse locally after breeding, especially females (Robinson et al. 2007b). Most juveniles captured during the autumn period are also likely to disperse away from their natal sites (Robinson et al. 2007b). Therefore, a large proportion of individuals captured in this study may be transients, i.e. individuals that are just 'passing through' the area and thus unlikely ever to be recaptured, and failure to account for transient individuals may negatively bias the survival estimates (Pradel et al. 1997). To overcome this bias, relative age models (Pollock 1975, Brownie and Robson 1983) were developed in a multi-state framework to estimate separately 'survival' of newly-marked birds (of which a substantial proportion are transients) and survival of birds recaptured at least once following initial marking ( = 'resident' birds; Prévot-Julliard et al. 1998). In practice, this approach works by constructing models in which survival over the interval immediately following the first (initial) capture $\left(\phi_{1}\right)$ differs from survival over subsequent intervals $\left(\phi_{2}\right)$. In addition, the ratio $\phi_{1} / \phi_{2}$ can be used to estimate the resident probability of newlymarked birds $\left(\zeta=\phi_{1} / \phi_{2}\right)$ and its counterpart, the transient probability $(1-\zeta)$ (Miller et al. 2003). In the present study, the initial capture of first-year birds (juveniles) were only possible during the autumn capture-recapture sessions, i.e. the spring (March-April) capture sessions were too early for any recently-fledged birds to be mist-netted. As a result, transient models allowed juveniles captured during their hatching year to become part of the pool of 'resident' birds only if they were recaptured during the following spring capture-recapture season or later. Cetti's warblers reach adult breeding propensity during their second calendar year, hence survival estimates of 'resident' birds in this paper refer to adult birds only.
The a priori set of competing models was developed based on biological reasoning and hypotheses to test. We had insufficient data to allow full time-specificity in survival which was permitted in the most general model instead to vary by year, sex and mass class, while both transition and capture probabilities were allowed to vary by season (spring-summer vs autumn-winter), sex, mass class and site (notation: $\left\{\phi_{\text {(year } \times \text { sex } \times \text { mass class) }}, \psi_{\text {(season } \times \operatorname{sex} \times \text { mass class } \times \text { site) }}\right.$, $\left.\mathrm{p}_{(\text {season } X \text { sex } X \text { mass class } X \text { site) }}\right\}$ where ' $X$ ' indicates a relationship that includes interaction terms). A set of candidate models (Table 1) was developed by modifying the structure of the general model. This included models with and without a transient effect on survival, models with additive effects (notation ' + ') between terms, and models with constant or seasonal variation in survival only. We also fitted models where season, sex, site and/or mass class effects were partially or entirely removed from the survival, transition or recapture components. Thus, as an example of notation, the model $\left\{\phi_{(\text {(season }+ \text { sex) }}, \psi_{\text {(mass class } \times \text { season) }}, \mathrm{P}_{(\text {site) }}\right\}$ corresponds to the reduced-parameter model in which survival differs only between sexes and season (with additive effect), transition rates differ according to mass class (i.e. $\psi_{\text {Light } \rightarrow \text { Heavy }} \neq \psi_{\text {Heavy }}$ $\rightarrow$ Light $)$ and season (with interaction), and recapture probabilities vary by site (Taipal vs Madriz). It can be also noted from this example that models that do not incorporate a mass class effect on transition probabilities would correspond to the case where the transition probabilities $\psi_{\text {Light } \rightarrow}$ Heavy $=\psi_{\text {Heavy } \rightarrow \text { Light }}$.

Data analyses were performed using the package RMark (Laake and Rexstad 2008) as an interface to Mark (White and Burnham 1999). During model development, capture probability was considered as a nuisance parameter and was modelled first, followed by transition probabilities. Survival was the last variable modelled because the main interest in this study was on the factors (i.e. season, sex and mass effects) potentially affecting adult survival. During this stepwise progression, parameters that had not yet been a focus of modelling were allowed to be as general as possible (cf. general model structure above). Akaike's information criterion corrected for small sample size (AIC) was applied to select the most parsimonious model(s) among the set of competing models (Burnham and Anderson 2002). $\mathrm{AIC}_{c}$ weights were also used to provide a relative measure of how well a model supports the data compared to other models (Burnham and Anderson 2002). A $\chi^{2}$ goodness-of-fit (GOF) test as available for the JollyMove (JMV) model (Pradel et al. 2003) was performed in program U-CARE (Choquet et al. 2005).

\section{Results}

\section{Data set}

Despite some slight differences between the two sexes, the annual cycle of variation in mean body mass reveals a common pattern for male and female cetti's warblers mist-netted at two sites in central Portugal from 2000 to 2010 (Fig. 1): mean mass peaked in March-April (onset of the breeding season), then progressively decreased in May-June-July (chick-rearing period) to reach its lowest value in SeptemberOctober (end of the moulting period). An upward trend in 
Table 1. Set of candidate models used to investigate apparent survival probability $(\phi)$, mass-state transition probability $(\psi)$ and recapture rates (p) in the cetti's warbler Cettia setti, central Portugal, 2000-2010. $\phi^{*}=$ transience effect on survival (i.e. first recapture interval following initial capture is modelled separately from all subsequent recapture periods). Subscripts in model notation are: 'Season' (spring-summer vs autumn-winter), 'Sex' (male vs female), 'Mass class' (light [female: 9.0-10.4 g, male: 12.5-13.9 g] vs heavy [female: 10.5-11.9 g, male: 14.0-16.0 g] individuals), and 'Site' (Madriz vs Taipal). Models with constant and year-specific parameters were also fitted to the data. $(\times)$ and $(+)$ denote interaction and additive (parallel) effect between terms, respectively.

\begin{tabular}{|c|c|c|c|c|c|c|}
\hline $\begin{array}{l}\text { Model } \\
\text { no. } \\
\end{array}$ & Model & $\mathrm{AIC}_{\mathrm{c}}$ & $\triangle \mathrm{AIC}_{\mathrm{c}}$ & $\begin{array}{c}\text { AIC } \\
\text { weight }\end{array}$ & $\begin{array}{c}\text { No. of } \\
\text { parameters }\end{array}$ & Deviance \\
\hline 1 & $\phi_{(\text {season) }}^{*} \psi_{\text {(season } \times \text { mass class) }} p_{(\text {site) }}$ & 1290.00 & 0 & 0.659 & 10 & 751.52 \\
\hline 2 & $\phi^{*}{ }_{(\text {season } \times \text { sex) }} \psi_{\text {(season } \times \text { mass class) }} p_{(\text {(site) }}$ & 1291.88 & 1.88 & 0.257 & 14 & 745.40 \\
\hline 3 & $\phi_{(\text {season } \times \text { sex })}^{*} \psi_{(\text {(season } \times \text { mass class) }} p_{(\text {see } \times \text { site) }}$ & 1294.15 & 4.15 & 0.082 & 16 & 743.68 \\
\hline 4 & $\phi_{\text {(season } \times \text { sex) }} \psi_{\text {(season } \times \text { mass class) }} p_{(\text {site) }}$ & 1309.49 & 19.49 & 0.00 & 10 & 771.01 \\
\hline 5 & $\phi_{\text {(season) }} \Psi_{\text {(season } \times \text { mass class) }} p_{\text {(site) }}$ & 1310.86 & 20.86 & 0.00 & 8 & 776.38 \\
\hline 6 & $\phi_{(\text {season } \times \text { sex })} \Psi_{(\text {season } \times \text { mass class })} p_{(\text {sex } \times \text { site })}$ & 1311.00 & 21.00 & 0.00 & 12 & 768.51 \\
\hline 7 & $\phi_{(\text {season } \times \text { mass class })} \Psi_{(\text {season } \times \text { mass class) }} \mathrm{p}_{(\text {site })}$ & 1314.77 & 24.77 & 0.00 & 10 & 776.29 \\
\hline 8 & $\phi_{\text {(season) })} \psi_{\text {(season } \times \text { mass class) }} p_{(\text {sex } \times \text { mass class })}$ & 1317.16 & 27.16 & 0.00 & 10 & 778.69 \\
\hline 9 & $\phi_{\text {(season) }} \Psi_{\text {(season } \times \text { mass class) }} p_{\text {(mass class) }}$ & 1328.69 & 28.69 & 0.00 & 8 & 795.21 \\
\hline 10 & $\phi_{(\text {season } \times \text { sex })} \Psi_{(\text {sex } \times \text { mass class })} p_{(\text {site })}$ & 1335.83 & 45.83 & 0.00 & 10 & 797.35 \\
\hline 11 & $\phi_{(\text {season) }} \Psi_{(\text {sex } \times \text { mass class })} \mathrm{p}_{(\text {site) }}$ & 1337.19 & 47.19 & 0.00 & 8 & 802.71 \\
\hline 12 & $\phi_{\text {(season } \times \text { sex } \times \text { mass class) }} \psi_{(\text {season } \times \text { mass class) }} p_{(\text {site })}$ & 1337.50 & 47.50 & 0.00 & 13 & 793.02 \\
\hline 13 & $\phi_{\text {(season } \times \text { sex) }} \psi_{\text {(mass class } \times \text { site) }} p_{(\text {(site) }}$ & 1339.59 & 49.59 & 0.00 & 10 & 801.11 \\
\hline 14 & $\phi_{\text {(season) }} \Psi_{\text {(site) }} \mathrm{p}_{\text {(site } \times \text { sex) }}$ & 1340.25 & 50.25 & 0.00 & 8 & 805.77 \\
\hline 15 & $\phi_{(\text {season } \times \text { sex) }} \Psi_{(\text {(site) }} p_{\text {(site) }}$ & 1340.78 & 50.78 & 0.00 & 8 & 806.31 \\
\hline 16 & $\phi_{\text {(year } \times \text { sex } \times \text { mass class })} \psi_{\text {(season } \times \text { sex } \times \text { mass class) }} p_{\text {(sex } \times \text { mass class } \times \text { site })}$ & 1341.60 & 51.60 & 0.00 & 60 & 703.12 \\
\hline 17 & $\phi_{\text {(season }+ \text { sex) }} \psi_{\text {(season }+ \text { mass class) }} p_{(\text {(site) }}$ & 1342.75 & 52.75 & 0.00 & 8 & 808.26 \\
\hline 18 & $\phi_{(\text {season } \times \text { sex })} \psi_{(\text {mass class })} p_{(\text {site })}$ & 1344.61 & 54.61 & 0.00 & 8 & 810.13 \\
\hline 19 & $\phi_{(\text {season } \times \text { mass class) }} \Psi_{(\text {site) }} \mathrm{p}_{(\text {site) }}$ & 1345.23 & 55.23 & 0.00 & 8 & 810.74 \\
\hline 20 & $\phi_{(\text {season) }} \psi_{(\text {mass class) }} \mathrm{p}_{\text {(site) }}$ & 1345.98 & 55.98 & 0.00 & 6 & 815.49 \\
\hline 21 & $\phi_{\text {(season) }} \psi_{\text {(mass class) }} p_{\text {(sex) }}$ & 1355.76 & 65.76 & 0.00 & 6 & 825.28 \\
\hline 22 & $\phi_{(\text {sex })} \psi_{\text {(mass class) }} \mathrm{p}_{(\text {(site) }}$ & 1363.38 & 73.38 & 0.00 & 6 & 832.90 \\
\hline 23 & $\phi_{\text {(season) }} \Psi_{\text {(mass class) }} p_{\text {(constant) }}$ & 1364.03 & 74.03 & 0.00 & 5 & 835.55 \\
\hline 24 & $\phi_{\text {(constant) }} \Psi_{\text {(mass class) }} p_{(\text {site) }}$ & 1365.22 & 75.22 & 0.00 & 5 & 836.74 \\
\hline 25 & $\phi_{\text {(season) }} \psi_{\text {(mass class) }} \mathrm{p}_{\text {(season) }}$ & 1365.91 & 75.91 & 0.00 & 6 & 847.43 \\
\hline 26 & $\phi_{\text {(year) }} \psi_{\text {(mass class) }} \mathrm{p}_{\text {(site) }}$ & 1371.33 & 81.33 & 0.00 & 15 & 822.84 \\
\hline
\end{tabular}

mean mass was subsequently apparent during the winter months, from November to January (Fig. 1).

Only those individuals weighed and measured (wing length) in March-April (high mean body mass) and September-October (low mean body mass) were incorporated in subsequent analyses, which included a total of 649 individuals: 394 females and 109 males mist-netted at Taipal and 91 females and 55 males caught at Madriz. Before running our multi-state models, we checked whether or not there was a systematic variation in body mass with structural size (as expressed by wing length), by means of linear regression analyses. In both sexes, the fit of the regression lines was very poor $\left(R^{2}=0.05\right.$ and 0.06 in females and males, respectively) despite a significant trend (both $F$-test with $\mathrm{p}<0.001$ ), which was mainly attributable to the high sample sizes involved in the analyses. Inter-individual differences in body mass in this dataset was thus likely to reflect inter-individual differences in fat/energy reserves, with no need to be adjusted according to structural size before inclusion in the modelling.

\section{Sample size and goodness-of-fit test}

The modelling was based on a total of 1122 capturerecaptures. The oldest cetti's warbler included in the models was a seven-year old female ringed as juvenile at Taipal in autumn 2003 and recaptured five times at the same site between spring 2004 and autumn 2010. The GOF test provided no evidence for lack of fit $\left(\chi^{2}{ }_{17}=11.65, \mathrm{p}=0.82\right)$, suggesting that the global model fitted the data adequately.

\section{Modelling recapture probability}

Models with constant or seasonal variation in recapture probabilities (models 25, 23, Table 1) were much less supported by the data than models in which this parameter varied according to either sex (model 21, Table 1) or site (model 20 , Table 1). Models with recapture probability modelled as a function of mass-state were also less supported compared to those with sex-specific or site-specific recapture probabilities (e.g. model 9 vs model 8 and model 9 vs model 5, Table 1). In fact, our top-ranked models (Table 2) suggested that the chances of recapture were nearly twice as high at the small wetland site (Madriz; $\mathrm{p}=0.371 \pm[\mathrm{SE}]$ 0.045) than at the larger site (Taipal; $\mathrm{p}=0.208 \pm 0.035$ ). In addition, females were less frequently mist-netted than males, especially at Taipal (see model 3 in Table 2 for such estimates).

\section{Modelling transition probability}

Modelling transition probabilities as a function of massstate variable alone was not a good fit when compared to those models incorporating additional effects (e.g. model 18 vs models 13, 10 and 4, Table 1 ). The best competing models were obtained by modelling transition probabilities in relation to both mass-state and season with interaction, 
Table 2. Survival and recapture estimates derived from the three best $\mathrm{AIC}_{\mathrm{c}}$-ranked models in Table 1. Recapture seasons are spring (March-April) and autumn (September-October) each year (2000-2010). The study was conducted at two distinct sites (site 1: Madriz; site 2: Taipal) in central Portugal.

\begin{tabular}{lccc}
\hline & & Estimate \pm SE & \\
\cline { 2 - 4 } Parameter & $\begin{array}{c}\text { Model 1 } \\
\text { (AIC-weight: 0.659) }\end{array}$ & $\begin{array}{c}\text { Model 2 } \\
\text { (AIC-weight: 0.257) }\end{array}$ & $\begin{array}{c}\text { Model 3 } \\
\text { (AIC-weight: 0.082) }\end{array}$ \\
\hline Adult male survival (spring-summer) & $0.787 \pm 0.177$ & $0.526 \pm 0.194$ & $0.536 \pm 0.184$ \\
Adult male survival (autumn-winter) & $0.371 \pm 0.097$ & $0.580 \pm 0.022$ & $0.566 \pm 0.202$ \\
Adult female survival (spring-summer) & $0.787 \pm 0.177$ & $0.999 \pm 0.001$ & $0.999 \pm 0.001$ \\
Adult female survival (autumn-winter) & $0.371 \pm 0.097$ & $0.278 \pm 0.060$ & $0.279 \pm 0.060$ \\
Recapture probability of females (site 1: Madriz) & $0.371 \pm 0.045$ & $0.371 \pm 0.045$ & $0.349 \pm 0.062$ \\
Recapture probability of males (site 1: Madriz) & $0.371 \pm 0.045$ & $0.371 \pm 0.045$ & $0.399 \pm 0.065$ \\
Recapture probability of females (site 2: Taipal) & $0.208 \pm 0.035$ & $0.208 \pm 0.035$ & $0.183 \pm 0.038$ \\
Recapture probability of males (site 2: Taipal) & $0.208 \pm 0.035$ & $0.208 \pm 0.035$ & $0.286 \pm 0.078$ \\
\hline
\end{tabular}

aSurvival estimates are for 'resident' birds (i.e. after accounting for the presence of transient individuals in the dataset).

rather than additive, effects (e.g. model 4 vs model 17, Table 1). Parameter estimates ( \pm SE) of models with lowest AIC (models 1, 2, 3 in Table 1) suggested that during springsummer cetti's warblers switching mass-class had a $56 \%$ probability to transit from heavy to light mass-class, while in autumn-winter, birds switching mass-class had a $70 \%$ probability to transit from light to heavy mass-class; Fig. 2 ). It was noteworthy that none of the birds included in the present analysis showed a transition from heavy to light mass-states in autumn-winter (Fig. 2).

\section{Modelling survival probability}

Models with either year-specific or constant survival probabilities were not well-supported by the data compared to those with a 'seasonal' effect (e.g. models 26, 24 vs model 20, Table 1). Models with survival influenced by 'sex' only were not a good fit (e.g. model 22 vs model 20 , Table 1) but those with an interaction between season and sex received substantial support from the data (e.g. model 10 vs model 11 and model 5 vs model 4, Table 1). Inclusion of mass-class as an additional effect did not improve model fit (e.g. models 7, 12 vs model

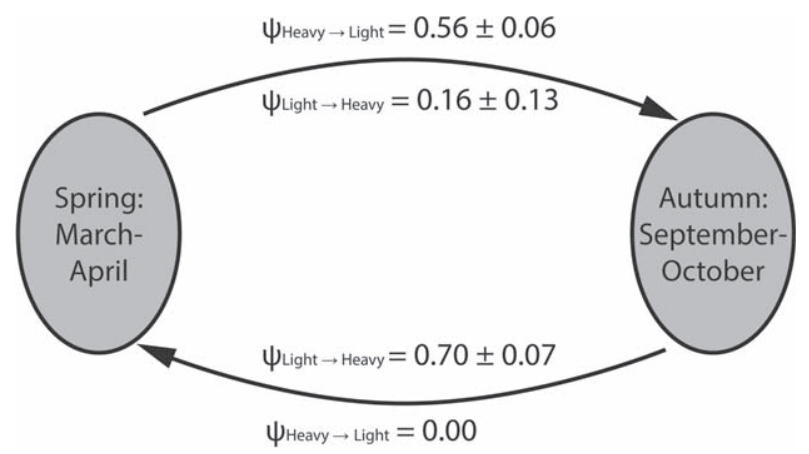

Figure 2. Seasonal transition probabilities $( \pm$ SE) between massclass states derived from models 1, 2, 3 in Table 1. Details of classes are light individuals (females: 9.0-10.4 g, males: 12.5-13.9 g) and heavy individuals (females: 10.5-11.9 g, males: 14.0-16.0 g). Capture-recapture seasons are spring (March-April) and autumn (September-October) each year (2000-2010). Note that the parameter estimate for the transition $\psi_{\text {Heavy } \rightarrow \text { Light }}$ between autumn and spring is on a boundary, so no SE is available.
5, Table 1). In fact, variation in survival was largely explained by factor 'season' in this dataset since removal of this effect from any of the competing models would result in a large increase in $\mathrm{AIC}_{c}$ values (e.g. model 4 vs model 22, Table 1). Finally, models accounting for the presence of transient birds provided the best explanation of the data as judged by $\mathrm{AIC}_{c}$-ranking (models $1,2,3$ vs models 4, 5, 6, Table 1). Average estimates of transient probability $(1-\zeta)$ from these three competing models (models 1,2,3, Table 1) suggested that most birds ringed in autumn (September-October) were transients in this population $(1-\zeta=0.809$ in males and 0.862 in females). A greater proportion of birds ringed in spring (March-April) were residents $(1-\zeta=0.533$ in males and 0.663 in females) when compared to autumn.

Survival estimates of resident birds derived from the top-ranked model (model 1, Table 1) suggested that adult survival was twice as high in spring-summer than in autumn-winter (model 1, Table 2). This top model was nonetheless followed by two closely-competing models that included a 'sex' effect on survival (models 2, 3, Table $1,2)$, so apparent adult survival estimates $( \pm \mathrm{SE})$ were derived from models $1,2,3$ using the averaging option in MARK (Fig. 3). These final estimates suggested that resident females in this population exhibited higher survival probability than resident males during the spring-summer season $\left(\phi_{\mathrm{RF}}=0.857 \pm 0.117\right.$ and $\left.\phi_{\mathrm{RM}}=0.698 \pm 0.181\right)$ while during the autumn-winter season, resident males survive better than resident females $\left(\phi_{\mathrm{RM}}=0.440 \pm 0.086\right.$ and $\phi_{\mathrm{RF}}=0.339 \pm 0.084$, Fig. 3).

\section{Discussion}

In the present study, we analysed an 11-yr ringing dataset of cetti's warblers obtained at two national ringing stations in central Portugal. In particular, we sought to understand 1) the pattern of body mass change during the annual cycle, 2) the role of mass-class in explaining individual survival, and 3) the role of sex in explaining individual survival in this polygynous species with a high sizedimorphism between males and females. Several predictions based on life-history theory and other available studies were made, and these are discussed below at the light of our modelling results. 


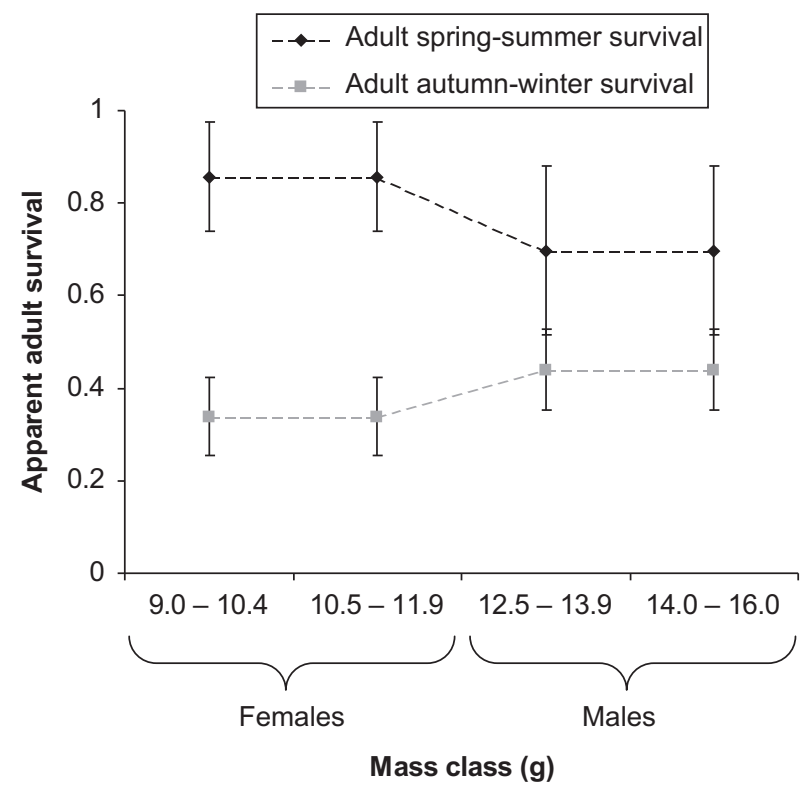

Figure 3. Apparent adult survival estimates $( \pm$ SE) derived from models 1, 2, 3 in Table 1 using the averaging option in MARK. Details of classes are light individuals (females: 9.0-10.4 g, males: $12.5-13.9 \mathrm{~g}$ ) and heavy individuals (females: $10.5-11.9 \mathrm{~g}$, males: $14.0-16.0 \mathrm{~g})$.

\section{Recapture probabilities}

In most mark-recapture studies, all survivors present in the study area cannot be detected at each sampling occasion. Consequently, the modelling of the recapture history dataset is needed to account for imperfect detection probabilities (i.e. detection probability $<1$ ) thereby yielding unbiased survival estimates (Williams et al. 2002). This situation is further highlighted by the low recapture rates achieved in the present study (all models with $\mathrm{p}<0.4$ ). Despite a similar mistnetting effort at both sites to meet the requirements of the Portuguese constant effort site scheme (i.e. equal no. of nets and re-trapping hours at both sites), our modelling detected a recapture rate at Taipal that was half that at Madriz, which seems consistent with the fact that cetti's warblers can disperse over a much wider area at Taipal (233 ha) compared with Madriz (89 ha). In addition, our modelling detected a sex-specific effect on recapture rates (model 3 ), a pattern that should be connected to behavioural differences between the sexes. For example, during the nesting season, males are very active in defending and moving over large breeding territories (Cramp and Brooks 1992), while polygynously-mated females establish smaller territories within their male's territory and defend them against other females mated with the same partner (Bibby 1982). Cetti's warbler females are also particularly discreet during the incubation period and forage only over their small territories during the chick-rearing period (Bibby 1982). Thus, altogether, the limited spatial movements of females during the breeding season should make them less susceptible to capture than males (Amrhein et al. 2012).

\section{Mass-class transition probabilities}

Our prediction that body mass varies seasonally was supported by the dataset. The actual pattern of body mass over the whole year suggested that mean body mass was usually high at the onset of the breeding season (March-April), but decreased thereafter and during the moulting period (Fig. 1). In fact, our models indicated that cetti's warblers had a $56 \%$ probability to transit from heavy to light mass-class between spring (March-April) and autumn (September-October; Fig. 2). The opposite pattern was apparent between autumn and spring with a tendency to gain mass between both sampling periods (i.e. birds showed a $70 \%$ probability to transit from light to heavy mass-class; Fig. 2). Previous studies in monogamous species have reported mass losses in passerines during the breeding season (Freed 1981, Suarez et al. 2005), especially during the chick-rearing period (Boyle et al. 2012). Our expectation was that mass loss during breeding might be mainly apparent in cetti's warbler females that, in common with other polygynous species, have a much higher parental investment than males (Bibby 1982). Sex-specific differences in the pattern of mass loss were, however, not supported by our data (model 8 with $\Delta \mathrm{AIC}_{c}>45$ units), indicating comparable energetic costs and/or strategies during reproduction in both males and females. Several reasons can be advanced to explain mass loss in both sex during breeding, including an energetic cost of reproduction (Bryant 1979, 1988, Nur 1988) or an adaptive response by which adults reduce their costs of flight during the rearing-period, thereby leading to an increase in the quantity of food available to their nestlings (Cichon 2001, Boyle et al. 2012). Mass loss in cetti's warbler males might also reflect energetic costs associated with defending their large territories during the breeding season. Previous experiments with small passerines have also reported decreasing fat levels and body mass during moult (Newton and Dawson 2011), although individual responses may differ according to factors such as sex and age (Gosler 1994). Our study suggests that cetti's warbler adults may incur a significant mass reduction during their complete post-breeding moult (Cramp and Brooks 1992), presumably because moulting is an energetically-demanding phase during their annual cycle. Conversely, individuals in both sexes had a high propensity to regain mass in autumn-winter. Winter fat storage has been strongly correlated with body mass in resident passerine species (Gosler 1996), so this seasonal change in body mass seems consistent with a winter fattening pattern (Macleod et al. 2005). In resident bird species, body mass is maintained at a higher level in winter compared with spring-summer, mainly as a response to increased starvation risk due to higher unpredictability of food resources and higher energetic requirements to survive longer/cooler nights (MacNamara and Houston 1990, Newton 1998, Macleod et al. 2005). Therefore, we speculate that resident cetti's warblers have a slight tendency to regain mass in autumn-winter in order to anticipate the reduced feeding opportunities they might encounter during midwinter (Lima 1986). We have no direct evidence of food reduction in winter at the study sites, but it can be noted that in central Portugal, most insectivorous passerines are summer visitors migrating to Africa in September-October, presumably as a response to the reduction in food availability at their breeding grounds.

\section{Survival probabilities}

Our top model (model 1) suggested that mortality is greatest during the non-breeding (autumn-winter) season, presumably 
because cetti's warblers depend for their survival on a constant access to food resources, which are most unpredictable during winter when adult bird mortality is thus expected to peak (Peach et al. 1999, Robinson et al. 2004, 2010). Contrary to our expectations, however, we did not find that heavier birds had a greater survival probability than lighter ones during winter (model 7 with $\Delta \mathrm{AIC}_{c}>24$ units). The absence of a relationship between mass-class and adult survival has also been reported for other species such as the European blackbird Turdus merula in central Italy (Miller et al. 2003). In this study, Miller et al. (2003) suggested that the relatively mild climatic conditions prevailing in their study area could explain the lack of a significant effect of body mass on survival. In fact, if carrying large amounts of fat, and hence being heavy, is advantageous to maximize fitness, we would expect this pattern to be more easily detected in populations living at northernmost latitudes (Lima 1986), where winter conditions can be much harsher than in southern Europe, such as in central Portugal (this study: minimum temperature recorded at closest weather station to our study area ranged $5-8^{\circ} \mathrm{C}$; <www. windguru.cz/fr/index.php?sc $=827>$ ) or central Italy (Miller et al. 2003). As further evidence for this, Gosler (1996) found a negative relationship between survival and body mass/fat reserves in great tits Parus major from UK during winters when food availability was low, but failed to detect any such pattern during milder years when winter food was apparently more abundant. Considering additional factors such as a bird's social status (i.e. dominant vs subdominant bird) is also important to fully understand the effect of body mass on survival (Gosler 1996), but it should be acknowledged that our study protocol did not allow for such a separation between marked individuals.

A peculiar life-history trait of regular polygynous passerines, including the cetti's warbler, is the strong sexual size-dimorphism evolved as a response to sexual selection, with the largest males being those that mate with the highest number of females (Bibby 1982). In such mating systems, 'stabilizing selection' might occur as a mechanism to prevent further increase in male size (Searcy and Yasukawa 1981). Therefore, Searcy and Yasukawa (1981) predicted that male size might be limited by a survival selection where the largest and heaviest males have lower survival rates, a pattern that was nonetheless not supported by our results. Note, however, that we had insufficient data to assign cetti's warbler males to more than two mass-classes, perhaps making the detection of a stabilizing selection in males difficult. Further studies should seek to examine the role of body mass in explaining survival rates when individuals are grouped in more than two mass-classes (e.g. three or more mass-classes including light, intermediate and heavy males).

Our initial prediction that males and females may incur different fitness costs (particularly during the breeding season) was supported by the data. In fact, our modelling results pointed towards an interaction between factors 'sex' and 'season' (Fig. 3): during the breeding/moulting seasons, resident females had a tendency to survive better than resident males ( 0.86 vs 0.70 , respectively) while during the non-breeding season (autumn-winter), the latter survived better than the former ( 0.44 vs 0.34 , respectively). Combining these seasonal estimates yields a similar annual survival rate of ca 0.30 in both sexes $(0.86 \times 0.34$ and $0.70 \times 0.44)$. This ad hoc annual survival estimate is slightly lower than that reported for many European species of similar size (see Siriwardena et al. 1998 for survival rates of 31 different passerines) but is nonetheless in line with estimates reported for European warblers breeding in wetlands such as the sedge Acrocephalus schoenobaenus (Siriwardena et al. 1998) and reed A. scirpaceus (Thaxter et al. 2006) warblers. The modelling approach developed here only permitted the estimation of 'apparent' survival rates (Williams et al. 2002), so the above estimates may also reflect a fair amount of permanent emigration from our study area. This also suggests that our sex-specific survival estimates may not represent a true pattern if, for instance, one sex has a much higher dispersal propensity than the other. In common with many other species, females are the more dispersive sex in the cetti's warbler (Robinson et al. 2007b), which could explain their lower apparent survival during the non-breeding season compared with males. However, cetti's warbler females have a higher apparent survival rate than males during the breeding season, hence permanent emigration and dispersal may not be the sole factors accounting for the sex-specific variations reported here.

In many bird species, mortality rates are higher for females which suffer from high predation rates during both incubation (Post and Götmark 2006a in pied flycatchers Ficedula hypoleuca) and feeding of offspring (Post and Götmark 2006b in Eurasian blackbirds Turdus merula). However, this situation was not apparent here despite the reduced parental investment of cetti's warbler males (Bibby 1982). Instead, our survival estimates suggest a higher survival cost in cetti's warbler males during breeding. A possible explanation for this pattern may lie in higher predation levels on males by aerial predators, presumably because of their more conspicuous behaviour (i.e. during song display and territorial defence; Bibby 1982) compared to females. Behavioural studies have found that polygynous males spend more time per hour singing and advertising their territories than monogamous males (Pilastro et al. 2002 in the rock sparrow Petronia petronia), hence time exposure to aerial predators might increase substantially for cetti's warbler males compared to other monogamous species, thereby explaining their low survival rates during the breeding season. Previous work with long-lived animals have also demonstrated a greater competition for mates in polygynous species compared to monogamous species, leading to skewed mortality rates towards males in the former situation (see Gaillard et al. 2003 for several study cases with mammals). For instance, in the polygynous black grouse Tetrao tetrix, males have lower survival rates than females due to high predation rates during the lekking season (Angelstam 1984). We are, however, not aware of previous studies reporting any relationship between polygyny, male survival and predation risk in passerines.

The fitness advantage of cetti's warbler females over males during the breeding/moulting seasons seems overridden by their higher mortality rates during the non-breeding season, although the failure of our modelling approach to account for possible sex-specific dispersal patterns suggests that interpretation of these results should be cautious. Nevertheless, a higher dispersal propensity of females over males during the non-breeding season may expose the former to a greater mortality risk compared to the latter (Steifetten and Dale 2006). 
In the cetti's warbler, males usually stay around their breeding territory year-round (Monticelli et al. unpubl), which should promote high survival in winter. The lower survival of resident females during that period may be explained by intra-specific competition and dominance behaviour (Lundberg 1985), in which females may be excluded towards sub-optimal habitats and less profitable food patches by dominant males in winter when food is scarcer.

\section{Conclusion}

This study suggests that cetti's warblers follow the predicted pattern of body mass change during the annual cycle of most passerines, in which adults have a tendency to lose mass during the course of the breeding season and to gain mass in autumn-winter. Body mass is not a good predictor of individual survival, consistent with the hypothesis that minimum temperatures and winter conditions are probably too mild in central Portugal to be a major determinant of cetti's warbler population dynamics. Adult survival is more likely to be shaped by ecological and behavioural differences between males and females. Importantly, our modelling results highlight that further experimental and field studies aiming to identify the main factors shaping survival and individual fitness in polygynous species should be conducted during different phases of the annual cycle.

Acknowledgements - We thank all the ringers who helped with fieldwork. We are also grateful to J. D. Nichols for analytical advice during the modelling of this dataset and to R. Robinson and V. Paiva for their constructive comments on earlier versions of this paper. During this study, DM and PMA were supported by research grants provided by the Portuguese Foundation for Science and Technology (FCT grants SFRH/BPD/66672/2009 and SFRH/ BD/69238/2010, respectively).

\section{References}

Amrhein, V., Scaar, B., Baumann, M., Minéry, N., Binnert, J.-P. and Korner-Nievergelt, F. 2012. Estimating adult sex ratios from bird mist netting data. - Methods Ecol. Evol. doi: 10.1111/j.2041-210X.2012.00207.x

Angelstam, P. 1984. Sexual and seasonal differences in mortality of the black grouse Tetrao tetrix in boreal Sweden. - Ornis Scand. 15: $123-134$.

Bibby, C. J. 1982. Polygyny and the breeding ecology of the cetti's warbler Cettia cetti. - Ibis 124: 288-301.

Bibby, C. J. and Thomas, D. K. 1984. Sexual dimorphism in size, moult and movements of cetti's warbler Cettia cetti. - Bird Study 31: 28-34.

BirdLife International 2012. Important bird areas factsheet: Paul da Madriz and Paul do Taipal. - < www.birdlife.org > accessed 19-06-2012.

Birkhead, T. R. and Møller, A. P. 1995. Extra-pair copulation and extra-pair paternity in birds. - Anim. Behav. 49: 843-848.

Bocci, A., Canavese, G. and Lovari, S. 2010. Even mortality patterns of the two sexes in a polygynous, near-monomorphic species: is there a flaw? - J. Zool. 280: 379-386.

Boyle, W. A., Winkler, D. W. and Guglielmo, C. G. 2012. Rapid loss of fat but not lean mass prior to chick provisioning supports the flight efficiency hypothesis in tree swallows. - Funct. Ecol. 26: 895-903.
Brownie, C. and Robson, D. S. 1983. Estimation of time-specific survival rates from tag-resighting samples: a generalization of the Jolly-Seber model. - Biometrics 39: 437-453.

Bryant, D. M. 1979. Reproductive costs in the house martin (Delichon urbica). - J. Anim. Ecol. 48: 655-675.

Bryant, D. M. 1988. Energy expenditure and body mass changes as measures of reproductive costs in birds. - Funct. Ecol. 2: $23-34$.

Burnham, K. P. and Anderson, D. R. 2002. Model selection and multimodel inference: a practical information-theoretic approach, 2nd ed. - Springer.

Choquet, R., Reboulet, A. M., Lebreton, J.-D., Gimenez, O. and Pradel, R. 2005. U-CARE 2.2 User's manual. - CEFE, Montpellier, France.

Cichon, M. 2001. Body-mass changes in female collared flycatchers: state-dependent strategy. - Auk 118: 550-552.

Clobert, J., Perrins, C. M., McCleery, R. H. and Gosler, A. G. 1988. Survival rate in the great tit (Parus major) in relation to sex, age and immigration status. - J. Anim. Ecol. 57: $287-306$.

Covas, R., Brown, C. R., Anderson, M. D. and Brown, M. B. 2002. Stabilizing selection on body mass in the sociable weaver Philetairus socius. - Proc. R. Soc. B 269: 1905-1909.

Cramp, S. and Brooks, D. J. 1992. Handbook of the birds of Europe, the Middle East, and North Africa: the birds of the Western Palearctic, Vol. 6. Warblers. - Oxford Univ. Press.

Cresswell, W. 1998. Diurnal and seasonal mass variation in blackbirds Turdus merula: consequences for mass-dependent predation risk. - J. Anim. Ecol. 67: 78-90.

Donald, P. F. 2007. Adult sex ratios in wild bird populations. - Ibis 149: 671-692.

EURING 2010. The EURING Exchange Code 2000 Plus. - The European Union for Bird Ringing. Thetford, UK.

Freed, L. A. 1981. Loss of mass in breeding wrens: stress or adaptation? - Ecology 62: 1179-1186.

Gaillard, J.-M., Loison, A., Festa-Bianchet, M., Yoccoz, N. G. and Solberg, E. 2003. Ecological correlates of life span in populations of large herbivorous mammals. - In: Carey, J. M. and Tuljapurkar, S. (eds), Life span: evolutionary, ecological and demographic perspectives. Supplement to population and development review, vol. 29. The Population Council, New York, pp. 39-56.

Gimenez, O., Viallefont, A., Charmantier, A., Pradel, R., Cam, E., Brown, C. R., Anderson, M. D., Bomberger Brown, M., Covas, R. and Gaillard J.-M. 2008. The risk of flawed inference in evolutionary studies when detectability is less than one. - Am. Nat. 172: 441-448.

Gosler, A. G. 1994. Mass-change during moult in the great tit Parus major. - Bird Study 41: 146-154.

Gosler, A. G. 1996. Environmental and social determinants of winter fat storage in the great tit Parus major. - J. Anim. Ecol. 65: $1-17$.

Houston, A. I. and McNamara, J. M. 1993. A theoretical investigation of the fat reserves and mortality levels of small birds in winter. - Ornis Scand. 24: 205-219.

Johnson, D. M., Stewart, G. L., Corley, M., Ghrist, R., Hagner, J., Ketterer, A., McDonnel, B., Newsom, W., Owen, E. and Samuels, P. 1980. Brown-headed cowbird (Molothrus ater) mortality in an urban roost. - Auk 97: 299-320.

Laake, J. and Rexstad, E. 2008. RMark: an alternative approach to building linear models in MARK. - <www.phidot.org/ software/mark/docs/book/pdf/app_3.pdf $>$.

Lebreton, J.-D., Nichols, J. D., Barker, R. J., Pradel, R. and Spendelow, J. A. 2009. Modeling individual animal histories with multistate capture-recapture models. - In: Caswell, H. (ed.), Advances in ecological research, vol. 41. Academic Press, pp. 87-173. 
Lieske, D. J., Warkentin, I. G., James, P. C., Oliphant, L. W. and Espie, R. H. M. 2000. Effects of population density on survival in merlins. - Auk 117: 184-193.

Lima, S. L. 1986. Predation risk and unpredictable feeding conditions: determinants of body mass in birds. - Ecology 67: 377-385.

Lundberg, P. 1985. Dominance behaviour, body weight and fat variations, and partial migration in European blackbirds Turdus merula. - Behav. Ecol. Sociobiol. 17: 185-189.

Macleod, R., Barnett, P., Clark, J. A. and Cresswell, W. 2005. Body mass change strategies in blackbirds Turdus merula: the starvation-predation risk tradeoff. - J. Anim. Ecol. 74: 292-302.

McGowan, C. P., Hines, J. E., Nichols, J. D., Lyons, J. E., Smith, D. R., Kalasz, K. S., Niles, L. J., Dey, A. D., Clark, N. A., Atkinson, P. W., Minton, C. D. T. and Kendall, W. 2011. Demographic consequences of migratory stopover: linking red knot survival to horsehoe crab spawning abundance. - Ecosphere 2: $\operatorname{art} 69$.

McNamara, J. M. and Houston, A. I. 1990. The value of fat reserves and the tradeoff between starvation and predation. Acta Biotheor. 38: 37-61.

Miller, M. W., Aradis, A. and Landucci, G. 2003. Effects of fat reserves on annual apparent survival of blackbirds Turdus merula. - J. Anim. Ecol. 72: 127-132.

Moreno, J. 1989. Strategies of mass change in breeding birds. - Biol. J. Linn. Soc. 37: 297-310.

Naef-Daenzer, B., Widmer, F. and Nuber, M. 2001. Differential post-fledging survival of great and coal tits in relation to their condition and fledging date. - J. Anim. Ecol. 70: 730-738.

Newton, I. 1998. Population limitation in birds. - Academic Press.

Newton, I. and Dawson, A. 2011. Seasonal changes in moult, body mass and reproductive condition in siskins Carduelis spinus exposed to daylength regimes simulating different latitudes. - J. Avian Biol. 42: 22-28.

Nichols, J. D. and Kendall, W. L. 1995. The use of multi-state capture-recapture models to address questions in evolutionary ecology. - J. Appl. Stat. 22: 835-846.

Nichols, J. D., Sauer, J. R., Pollock, K. H. and Hestbeck, J. B. 1992. Estimating transition probabilities for stage-based population projection matrices using capture-recapture data. - Ecology 73: 306-312.

Nur, N. 1988. The cost of reproduction in birds: an examination of the evidence. - J. Anim. Ecol. 76: 155-168.

Peach, W. J., Siriwardena, G. M. and Gregory, R. D. 1999. Longterm changes in over-winter survival rates explain the decline of reed buntings Emberiza schoeniclus in Britain. - J. Appl. Ecol. 36: 798-811.

Pilastro, A., Griggio, M., Biddau, L. and Mingozzi, T. 2002. Extrapair paternity as a cost of polygyny in the rock sparrow: behavioural and genetic evidence of the 'tradeoff' hypothesis. - Anim. Behav. 63: 967-974.

Pollock, K. H. 1975. A $K$-sample tag-recapture model allowing for unequal survival and catchability. - Biometrika 62: 577-583.

Post, P. and Götmark, F. 2006a. Predation by sparrowhawks Accipiter nisus on male and female pied flycatchers Ficedula hypoleuca in relation to their breeding behaviour and foraging. - J. Avian Biol. 37: 158-168.

Post, P. and Götmark, F. 2006b. Foraging behavior and predation rish in male and female blackbirds Turdus merula during the breeding season. - Aule 123: 162-170.

Pradel, R., Hines, J. E., Lebreton, J.-D. and Nichols, J. D. 1997. Capture-recapture survival models taking account of transients. - Biometrics 53: 60-72.
Pradel, R., Wintrebert, C. M. A. and Gimenez, O. 2003. A proposal for a godness-of-fit test to the Arnason-Schwarz multisite capture-recapture model. - Biometrics 59: 43-53.

Prévot-Julliard, A.-C., Lebreton, J.-D. and Pradel, R. 1998. Re-evaluation of adult survival of black-headed gulls (Larus ridibundus) in presence of recapture heterogeneity. - Auk 115: 85-95.

Robinson, R. A., Green, R. E., Baillie, S. R., Peach, W. J. and Thomson, D. L. 2004. Demographic mechanisms of the population decline of the song thrush Turdus philomelus in Britain. - J. Anim. Ecol. 73: 670-682.

Robinson, R. A., Baillie, S. R. and Crick, H. Q. P. 2007 a. Weather-dependent survival: implications of climate change for passerine population processes. - Ibis 149: 357-364.

Robinson, R. A., Freeman, S. N., Balmer, D. E. and Grantham, M. J. 2007b. Cetti's warbler Cettia cetti: analysis of an expanding population. - Bird Study 54: 230-235.

Robinson, R. A., Kew, J. J. and Kew, A. J. 2010. Survival of suburban blackbirds Turdus merula varies seasonally but not by sex. - J. Avian Biol. 41: 83-87.

Sæther, B.-E., Engen, S. and Matthysen, E. 2002. Demographic characteristics and population dynamical patterns of solitary birds. - Science 295: 2070-2073.

Schubert, K. A., Mennill, D. J., Ramsay, S. M., Otter, K. A., Ratcliffe L. M. and Kraus, C. 2008. Between-year survival and rank transitions in male black-capped chickadees (Poecile atricapillus): a multistate modeling approach. - Auk 125: 629-636.

Searcy, W. A. and Yasukawa, K. 1981. Sexual size dimorphism and survival of male and female blackbirds (Icteridae). - Auk 98: 457-465.

Siriwardena, G. M., Baillie, S. R. and Wilson, J. D. 1998. Variation in the survival rates of some British passerines with respect to their population trends on farmland. - Bird Study 45: 276-292.

Steifetten, O. and Dale, S. 2006. Viability of an endangered population of ortolan buntings: the effect of a skewed operational sex ratio. - Biol. Conserv. 132: 88-97.

Suarez, F., Sanchez, A. M., Herranz, J., Traba, J. and Yanes, M. 2005. Parental body mass changes during the nesting stage in two lark species in a semi-arid habitat. - J. Arid Environ. 62: 45-54.

Svensson, L. 1992. Identification guide to European passerines. - Published privately, Stockholm, Sweden.

Thaxter, C. B., Redfern, C. P. F. and Bevan, R. M. 2006. Survival rates of adult reed warblers Acrocephalus scirpaceus at a northern and southern site in England. - Ringing Migration 23: 65-79.

Tinbergen, J. M., van Balen, J. H. and van Eck, H. M. 1985. Density-dependent survival in an isolated great tit population: Kluyver's data reanalysed. - Ardea 73: 38-48.

Villaran, A. 2000. Biometry, phenology and sex ratio of cetti's warbler Cettia cetti in reedbeds of the Tagus Basin (central Spain). - Butlleti del Grup Catala d'Anellament 17: 1-9.

Webster, M. S. 1991. Male parental care and polygyny in birds. - Am. Nat. 137: 274-280.

White, G. C. and Burnham, K. P. 1999. Program MARK: survival estimation from populations of marked animals. - Bird Study 46 (Suppl): 120-139.

Williams, B. K., Nichols, J. D. and Conroy, M. J. 2002. Analysis and management of animal populations. - Academic Press.

Witter, M. S. and Cuthill, I. C. 1993. The ecological costs of avian fat storage. - Phil. Trans. R. Soc. B 340: 73-92. 